\title{
Stable estimation of two coefficients in a nonlinear Fisher-KPP equation
}

\author{
Michel Cristofol ${ }^{1}$ and Lionel Roques ${ }^{2}$ \\ 1 Aix-Marseille Université, LATP, Faculté des Sciences et Techniques \\ Avenue Escadrille Normandie-Niemen, F-13397 Marseille Cedex 20, France \\ 2 UR 546 Biostatistique et Processus Spatiaux, INRA, F-84000 Avignon, France \\ E-mail: ${ }^{1}$ cristo@latp.univ-mrs.fr, ${ }^{2}$ lionel.roques@avignon.inra.fr
}

\begin{abstract}
We consider the inverse problem of determining two non-constant coefficients in a nonlinear parabolic equation of the Fisher-Kolmogorov-PetrovskyPiskunov type. For the equation $u_{t}=D \Delta u+\mu(x) u-\gamma(x) u^{2}$ in $(0, T) \times \Omega$, which corresponds to a classical model of population dynamics in a bounded heterogeneous environment, our results give a stability inequality between the couple of coefficients $(\mu, \gamma)$ and some observations of the solution $u$. These observations consist in measurements of $u$ : in the whole domain $\Omega$ at two fixed times; in a subset $\omega \subset \subset \Omega$ during a finite time interval; and on the boundary of $\Omega$ at all times $t \in(0, T)$. The proof relies on parabolic estimates together with parabolic maximum principle and Hopf's lemma which enable us to use a Carleman inequality. This work extends previous studies on stable determination of non-constant coefficients in parabolic equations, as it deals with two coefficients and with a nonlinear term. A consequence of our results is the uniqueness of the couple of coefficients $(\mu, \gamma)$, given the observation of $u$. This uniqueness result was obtained in a previous article but in the one-dimensional case only.
\end{abstract}

Keywords: Inverse Problem · Nonlinear parabolic equation · Two coefficients · Stability - Uniqueness · Carleman estimate 
Version définitive du manuscrit publiée dans / Final version of the manuscript published in :

Inverse problems (2013), 22 p., DOI: no data.

Stable estimation of two coefficients in a nonlinear Fisher-KPP equation

\section{Introduction}

Reaction-diffusion models of the form:

$$
u_{t}=D \Delta u+f(u), t>0, x \in \Omega \subset \mathbb{R}^{n},
$$

arise in several fields of application. These applications range from population dynamics $[1,2,3,4]$ and population genetics $[5,6,7,8,9]$ to chemistry [10, 11], and combustion [12].

Spatial heterogeneities can easily be incorporated in these models by modifying the diffusion and reaction terms. For instance, in population dynamics, several authors have considered the case of spatially heterogeneous reaction terms of the Fisher-KPP type: $f(x, u)=\mu(x) u-\gamma(x) u^{2}$. In this case, the model (1.1) becomes:

$$
u_{t}=D \Delta u+\mu(x) u-\gamma(x) u^{2}, t>0, x \in \Omega \subset \mathbb{R}^{n} .
$$

The model $\left(\mathcal{S}_{\mu, \gamma}\right)$ has been introduced by Skellam [13] in the context of population dynamics, and then studied in bounded domains $[14,15]$ and periodic environments [3, $16,17,18,19,20,21]$. In these references, the quantity $u(t, x)$ generally stands for a population density and the coefficients $D>0, \mu(x)$ and $\gamma(x)>0$ respectively correspond to the diffusion coefficient, the intrinsic growth rate coefficient (i.e., the birth rate minus the death rate in the absence of competition) and a coefficient measuring the effects of competition on the birth and death rates.

With the increasing frequency of biological invasions [22], ecologists and modelers are often faced with species invading a new environment. In such cases, the reproduction and dispersal features of the species in its new environment are often not known and have to be estimated [23]. For the reaction-diffusion model $\left(\mathcal{S}_{\mu, \gamma}\right)$, the precise estimation of the coefficients of the model is of critical importance, as the behavior of the model strongly depends on the value of the coefficients [14]. In particular, the success of an invasion [15] and the rate of spread of a successful invasion [19, 24] depend on the coefficients $D$ and $\mu(x)$, while the stationary state towards which the solution converges depends on $D, \mu(x)$ and $\gamma(x)$.

Parameter estimation for models based on differential equations often relies on mechanistic-statistical approaches [23, 25, 26], which use a statistical model for the observation process, and allow one to compute the likelihood of the parameters, or coefficients. However, depending on the model and on the set of observations, the coefficients may not always be identifiable. This means that different set of coefficients can lead to the same observations and therefore have the same likelihood. It is therefore important to derive conditions on the observations which guarantee that there is a unique set of coefficients leading to the observations. Since real observations are generally noisy, it is also important to check whether close observations lead to close estimations of the coefficients. The results of this paper give such conditions on the observations for the estimation of the parameters $\mu(x)$ and $\gamma(x)$ of $\left(\mathcal{S}_{\mu, \gamma}\right)$. In particular, we obtain a stability inequality of the form

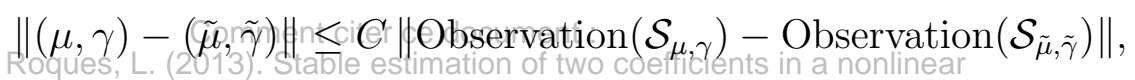


which links the distance between two sets of coefficients with the distance between two sets of observations. Such stability inequalities lead to the uniqueness of the coefficients $(\mu, \gamma)$ given the observation "Observation $\left(\mathcal{S}_{\mu, \gamma}\right)$ ". They are also useful for the numerical reconstruction of the coefficients using noise-free observations [27].

Similar stability results had already been obtained for a unique unknown coefficient $\mu(x)$ in the linear case $u_{t}=D \Delta u+\mu(x) u$. For such reaction-diffusion equations, the derivation of stability inequalities often relies on Carleman estimates [28, 29, 30, 31]. The idea of using Carleman estimates for solving such coefficient inverse problems has been introduced in the foundational paper [32]; this technique is therefore often called the "Bukhgeim-Klibanov method". The idea of obtaining Lipschitz stability estimates for coefficient inverse problems in parabolic equations was then proposed in [30] for the first time; see also the survey paper [33].

The solution of the linear equation $u_{t}=D \Delta u+\mu(x) u$ can blow up when $t \rightarrow \infty$, depending on the coefficients $D, \mu(x)$ and on the boundary conditions. The nonlinear reaction term $f(x, u)=\mu(x) u-\gamma(x) u^{2}$ that we consider in this paper is generally more realistic, at least in the context of population dynamics, since it always lead to bounded solutions (uniformly in time). With such a reaction term, uniqueness results for the coefficients $\mu(x), \gamma(x)$ have been proven recently in the one-dimensional case [34, 35] under the assumption that the density $u\left(t, x_{0}\right)$ and its spatial derivative $\frac{\partial u}{\partial x}\left(t, x_{0}\right)$ are known at a point $x_{0}$ and during a time interval $t \in(0, \varepsilon)$. Comparable results have also been obtained for systems of nonlinear parabolic equations [36]. However, the methods developed in [34, 35, 36], which do not rely on Carleman estimates, cannot be applied as such to higher dimensions $n \geq 2$, and do not lead to stability inequalities, even when $n=1$. Uniqueness and stability results for nonlinear but homogeneous (i.e., independent of $x$ ) reactions terms $f(u)$ can also be derived from boundary measurements $[37,38,39,40,41,42]$.

Here, our aim is to obtain a global stability inequality which enables to simultaneously estimate both coefficients $\mu(x)$ and $\gamma(x)$ of $\left(\mathcal{S}_{\mu, \gamma}\right)$, and to prove their uniqueness, given the following information: (i) $\mu(x)$ and $\gamma(x)$ are regular $\left(C^{5}(\bar{\Omega})\right)$ and are known near the boundary $\partial \Omega$; (ii) the density $u_{i}(x)=u(0, x)$ is known in $\Omega$ at $t=0$; (iii) $u$ is known on $\partial \Omega$ and satisfies Neumann boundary conditions in $[0, \infty) \times \partial \Omega$; (iv) the density $u(t, x)$ is known in a finite time interval and in a subset $\omega \subset \subset \Omega$; (v) the densities $u\left(t_{0}, x\right)$ and $u\left(t_{1}, x\right)$ are known at two fixed times $t_{0}, t_{1}$ and for all $x \in \Omega$. The main tools used to establish these new results are Carleman estimates with special weights and parabolic estimates together with parabolic maximum principle and Hopf's lemma.

\section{Hypotheses and main results}

In this study, we assume that $\Omega$ is a smooth and bounded domain. As mentioned in the Introduction Section, we assume that the unknown coefficients $\mu(x)$ and $\gamma(x)$ are 


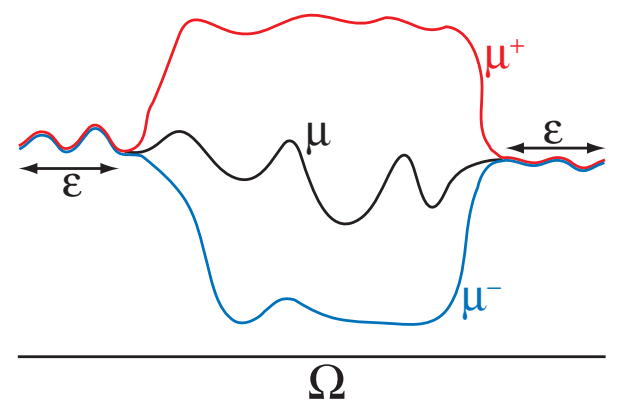

Figure 1. Schematic example of a function $\mu$ in $M$.

known near the boundary of $\Omega$, and bounded by known functions. In other terms,

$$
\mu(x) \in M:=\left\{\tilde{\mu} \in C^{5}(\bar{\Omega}) \text { s.t. } \mu^{-} \leq \tilde{\mu} \leq \mu^{+} \text {on } \bar{\Omega} \text { and }\|\tilde{\mu}\|_{C^{5}(\bar{\Omega})} \leq m\right\},
$$

and

$$
\gamma(x) \in \Gamma:=\left\{\tilde{\gamma} \in C^{5}(\bar{\Omega}) \text { s.t. } \gamma^{-} \leq \tilde{\gamma} \leq \gamma^{+} \text {on } \bar{\Omega} \text { and }\|\tilde{\gamma}\|_{C^{5}(\bar{\Omega})} \leq m\right\},
$$

for $m>0$ and four functions $\mu^{-}, \mu^{+}, \gamma^{-}, \gamma^{+}$in $C^{5}(\bar{\Omega})$ such that $0<\mu^{-} \leq \mu^{+}$and $0<\gamma^{-} \leq \gamma^{+}$on $\bar{\Omega}$ and

$$
\mu^{-}(x)=\mu^{+}(x) \text { and } \gamma^{-}(x)=\gamma^{+}(x) \text { if } d(x, \partial \Omega)<\varepsilon,
$$

for some positive constant $\varepsilon>0$. Here, $d(x, \partial \Omega)$ corresponds to the usual euclidian distance between any point $x \in \bar{\Omega}$ and the boundary of $\Omega$.

Let us fix a couple of coefficients $(\mu, \gamma)$ in $M \times \Gamma$. Our aim is to state a stability inequality which enables us to reconstruct these two coefficients based on some observations of the solution $u$ of:

$$
\left\{\begin{array}{l}
\partial_{t} u=D \Delta u+u(\mu-\gamma u) \text { in }(0, T) \times \Omega, \\
\partial_{\nu} u=0 \text { on }[0, T) \times \partial \Omega, \\
u(0, \cdot)=u_{i} \text { in } \Omega,
\end{array}\right.
$$

for some constants $D>0, T>0$ and some function $u_{i}$ in $C^{7}(\bar{\Omega})$.

As already mentioned in the Introduction Section, we assume that $u$ is known on $\partial \Omega$. Thus, the stability inequality has to link the distance between $(\mu, \gamma)$ and any set of coefficients $(\tilde{\mu}, \tilde{\gamma})$ in $M \times \Gamma$ with the distance between the observation of $u$ and the observation of the solution $\tilde{u}$ of

$$
\left\{\begin{array}{l}
\partial_{t} \tilde{u}=D \Delta \tilde{u}+\tilde{u}(\tilde{\mu}-\tilde{\gamma} \tilde{u}) \text { in }(0, T) \times \Omega, \\
\tilde{u}=u \text { on }[0, T) \times \partial \Omega, \\
\tilde{u}(0, \cdot)=u_{i} \text { in } \Omega .
\end{array}\right.
$$

Existence, uniqueness and regularity of the solution $u$ are classical (see e.g. [43]). In particular, the function $u$ belongs to $C_{1}^{2}([0, T) \times \bar{\Omega}) \ddagger$. Similarly, the problem (2.4) admits a unique solution $\tilde{u}$, which belongs to $C_{1}^{2}([0, T) \times \bar{\Omega})$ (see Lemma 3.1).

$\ddagger$ The spaces $C_{j}^{i}([0, T) \times \bar{\Omega})$ are spaces of functions on $[0, T) \times \bar{\Omega}$ whose derivatives up to order $i$ in $x$ and order $j$ in $t$ are continuous.ent citer ce document 
In order to state our stability inequality, we need other assumptions on $u_{i}$ :

$$
u_{i}>0 \text { on } \bar{\Omega}, \partial_{\nu} u_{i}=0 \text { on } \partial \Omega \text { and } 6 \max _{\bar{\Omega}} u_{i}<\min _{\bar{\Omega}} \mu^{-} / \max _{\bar{\Omega}} \gamma^{+},
$$

and $u_{i}$ is a subsolution of the problem (2.3), in the sense:

$$
-D \Delta u_{i}-u_{i}\left(\mu^{-}-\gamma^{+} u_{i}\right)<0 \text { on } \bar{\Omega} \text {. }
$$

Note that the set of initial conditions $u_{i}$ satisfying both assumptions (2.5)-(2.6) is not empty. For instance, we can take any constant lower than $\min _{\bar{\Omega}} \mu^{-} /\left(6 \max _{\bar{\Omega}} \gamma^{+}\right)$. Another type of non-constant initial conditions satisfying these assumptions are presented in Appendix A.

Before stating our main theorem, let us state a preliminary lemma which gives a Harnack-type inequality:

Lemma 2.1. It exists a bounded interval $\mathcal{T}$ in $(0, \infty)$ such that, for any couple $\left(t_{0}, t_{1}\right)$ with $0<t_{0} \leq \inf \mathcal{T}<\sup \mathcal{T} \leq t_{1}$, and for all $(\mu, \gamma)$ and $(\tilde{\mu}, \tilde{\gamma}) \in M \times \Gamma$,

$$
6 \max _{x \in \bar{\Omega}} \tilde{u}\left(t_{0}, x\right)<\min _{x \in \bar{\Omega}} \tilde{u}\left(t_{1}, x\right) .
$$

The interval $\mathcal{T}$ can be computed explicitly, see Appendix B for the proof of Lemma 2.1.

Our main result is:

Theorem 2.2. For any $\omega \subset \subset \Omega$, any time interval $\left(t_{0}, t_{1}\right)$ containing $\mathcal{T}$ and any couple $(\mu, \gamma)$ in $M \times \Gamma$, there exist $\delta \in\left(0, t_{0}\right)$ and a constant $C$ such that for all $\tilde{\mu} \in M, \tilde{\gamma} \in \Gamma$,

$$
\begin{gathered}
\|\mu-\widetilde{\mu}\|_{L^{2}(\Omega)}^{2}+\|\gamma-\widetilde{\gamma}\|_{L^{2}(\Omega)}^{2} \leq C G(u, \tilde{u}), \quad \text { with } \\
G(u, \tilde{u})=\|u-\widetilde{u}\|_{H^{2}\left(\left(t_{0}-\delta, t_{1}+\delta\right), L^{2}(\omega)\right)}^{2}+\left\|(u-\widetilde{u})\left(t_{0}, \cdot\right)\right\|_{H^{2}(\Omega)}^{2}+\left\|(u-\widetilde{u})\left(t_{1}, \cdot\right)\right\|_{H^{2}(\Omega)}^{2} .
\end{gathered}
$$

A straightforward corollary is a uniqueness result $(\mu, \gamma) \equiv(\tilde{\mu}, \tilde{\gamma})$, given $u\left(t_{0}, x\right)$, $u\left(t_{1}, x\right)$ for $x \in \Omega$ and $u(t, x)$ for $t \in\left(t_{0}-\delta, t_{1}+\delta\right)$ and $x \in \omega$. Another practical consequence of Theorem 2.2 is to allow a numerical reconstruction of the unknown coefficients $\mu$ and $\gamma$, given the partial measurements (i), (ii), (iii), (iv), (v) detailed at the end of the Introduction Section (see [27, 29]).

The paper is organized as follows. In Section 3 we prove regularity results and uniform estimates for the solution of (2.4). Then, in Section 4, we prove the stability inequality stated in Theorem 2.2 .

\section{Preliminary lemmas}

In this section, we derive estimates of the solution $\tilde{u}$ of (2.4), which are independent of the choice of the coefficients $\tilde{\mu}$ in $M$ and $\tilde{\gamma}$ in $\Gamma$. These estimates will be used in the proof of the stability inequality developed in Section 4.

We begin with the existence, uniqueness and regularity of the solution $\tilde{u}$ of (2.4). Cristofol, M., Roques, L. (2013). Stable estimation of two coefficients in a nonlinear 
Lemma 3.1. The problem (2.4) admits a solution $\tilde{u}$ in $C_{1}^{2}([0, T) \times \bar{\Omega})$, and this solution is unique.

Proof. Let us check that the boundary condition $\tilde{u}=u$ on $\partial \Omega$ and the initial condition $\tilde{u}(0, \cdot)=u_{i}$ in $\Omega$ satisfy the compatibility condition

$$
\partial_{t} \tilde{u}(0, x)=\partial_{t} u(0, x)=D \Delta u_{i}(x)+u_{i}(x)\left(\tilde{\mu}(x)-\tilde{\gamma}(x) u_{i}(x)\right) \text { on } \partial \Omega .
$$

Since $u \in C_{1}^{2}([0, T) \times \bar{\Omega})$ the equation $\partial_{t} u(t, x)=D \Delta u(t, x)+u(t, x)(\mu(x)-\gamma(x) u(t, x))$ in (2.3) remains valid at $t=0$ and for all $x \in \partial \Omega$. Thus, we have:

$$
\partial_{t} u(0, x)=D \Delta u_{i}(x)+u_{i}(x)\left(\mu(x)-\gamma(x) u_{i}(x)\right) \text { on } \partial \Omega .
$$

Since the couples $(\mu, \gamma)$ and $(\tilde{\mu}, \tilde{\gamma})$ belong to $M \times \Gamma$, we have $\mu(x)=\tilde{\mu}(x)$ and $\gamma(x)=\tilde{\gamma}(x)$ for all $x \in \partial \Omega$. Thus, the compatibility condition (3.7) is fulfilled. It follows from Theorems 8 and 9 in [44], Chap. 7, Sec. 4 that (2.4) admits a unique solution $\tilde{u}$, which belongs to $C_{1}^{2}([0, T) \times \bar{\Omega})$.

We then obtain lower bounds for $\tilde{u}$ and $\tilde{u}_{t}$, which are independent of the choice of $(\mu, \gamma)$ and $(\tilde{\mu}, \tilde{\gamma})$ :

Lemma 3.2. For any $T>0$,

(i) it exists $r_{1}>0$ such that,

$$
\text { for all }(\mu, \gamma) \text { and }(\tilde{\mu}, \tilde{\gamma}) \in M \times \Gamma, \tilde{u} \geq r_{1} \text { on }[0, T] \times \bar{\Omega} ;
$$

(ii) it exists $r_{2}>0$ such that,

$$
\text { for all }(\mu, \gamma) \text { and }(\tilde{\mu}, \tilde{\gamma}) \in M \times \Gamma, \tilde{u}_{t} \geq r_{2} \text { on }[0, T] \times \bar{\Omega} \text {. }
$$

Proof of (i) Let us set

$$
\underline{\mu}^{-}=\min _{\bar{\Omega}}\left\{\mu^{-}\right\}, \bar{\gamma}^{+}=\max _{\bar{\Omega}}\left\{\gamma^{+}\right\}, \text {and } \underline{u_{i}}=\min _{\bar{\Omega}}\left\{u_{i}\right\} .
$$

Let us then define $u^{-}$as the solution of the ODE:

$$
\frac{d u^{-}}{d t}=u^{-}\left(\underline{\mu}^{-}-\bar{\gamma}^{+} u^{-}\right), u^{-}(0)=\underline{u_{i}} .
$$

The function $(t, x) \mapsto u^{-}(t)$ is a subsolution of the equation satisfied by $u$, with Neumann boundary conditions and with $u^{-}(0) \leq u(0, x)$ on $\bar{\Omega}$. We therefore have

$$
u^{-}(t) \leq u(t, x) \text { for all } t \in[0, T], x \in \bar{\Omega} .
$$

Since the function $(t, x) \mapsto u^{-}(t)$ is also a subsolution of the equation satisfied by $\tilde{u}$, with $u^{-}(t) \leq u(t, x)=\tilde{u}(t, x)$ on $[0, T] \times \partial \Omega$, and since $\tilde{u}(0, x) \geq \underline{u_{i}}>0$ on $\bar{\Omega}$, we get:

$$
0<r_{10}: \overline{\bar{c}} \min _{t \in[0, T]} u_{c i}^{-}(t) \leq \tilde{u}(t, x) \text { on }[0, T] \times \bar{\Omega} \text {. }
$$


Proof of (ii) First, we show that $\partial_{t} u$ and $\partial_{t} \tilde{u}$ are classical (i.e. strong) solutions of some parabolic problem. Let $v$ be the classical solution of

$$
\left\{\begin{array}{l}
\partial_{t} v=D \Delta v+v(\mu-2 \gamma u) \text { in }(0, T) \times \Omega, \\
\partial_{\nu} v=0 \text { on }[0, T) \times \partial \Omega, \\
v(0, \cdot)=D \Delta u_{i}+u_{i}\left(\mu-\gamma u_{i}\right) \text { in } \Omega
\end{array}\right.
$$

Differentiating the equation satisfied by $u$ with respect to $t$, and using Theorems 4 and 5 in [45] Chap. 7, we obtain that $\partial_{t} u$ is the unique weak solution of (3.9). By uniqueness, it follows that $\partial_{t} u=v$ and therefore $\partial_{t} u$ is a classical solution of (3.9). Similarly, $\tilde{v}=\partial_{t} \tilde{u}$ is a classical solution of

$$
\left\{\begin{array}{l}
\partial_{t} \tilde{v}=D \Delta \tilde{v}+\tilde{v}(\tilde{\mu}-2 \tilde{\gamma} \tilde{u}) \text { in }(0, T) \times \Omega \\
\tilde{v}=v \text { on }[0, T) \times \partial \Omega \\
\tilde{v}(0, \cdot)=D \Delta u_{i}+u_{i}\left(\tilde{\mu}-\tilde{\gamma} u_{i}\right) \text { in } \Omega
\end{array}\right.
$$

Let us set

$$
\bar{\mu}^{+}=\max _{\bar{\Omega}}\left\{\mu^{+}\right\} \text {and } \overline{u_{i}}=\max _{\bar{\Omega}}\left\{u_{i}\right\}
$$

Let us then define $u^{+}$as the solution of the ODE:

$$
\frac{d u^{+}}{d t}=u^{+} \bar{\mu}^{+}, u^{+}(0)=\overline{u_{i}} \text {. }
$$

The function $(t, x) \mapsto u^{+}(t)$ is a supersolution of the equation satisfied by $u$, with Neumann boundary conditions and with $u(0, x) \leq u^{+}(0)$ on $\bar{\Omega}$. We therefore have

$$
u(t, x) \leq u^{+}(t) \leq \overline{u_{i}} e^{\bar{\mu}^{+} T} \text { for all } t \geq 0, x \in \bar{\Omega} .
$$

Let us consider the solution $v^{-}$of the ODE:

$$
\left\{\begin{array}{l}
\frac{d v^{-}}{d t}=v^{-}\left(\underline{\mu}^{-}-2 \bar{\gamma}^{+} e^{\bar{\mu}^{+} T}\right), \text { for } t \in(0, T), \\
v^{-}(0)=\min _{\bar{\Omega}}\left\{D \Delta u_{i}+u_{i}\left(\mu^{-}-\gamma^{+} u_{i}\right)\right\} .
\end{array}\right.
$$

From the assumption (2.6), we know that $v^{-}(0)>0$. It easily follows from CauchyLipschitz theorem that $v^{-}(t)>0$ for all $t \in[0, T]$. We define

$$
0<r_{2}:=\min _{t \in[0, T]} v^{-}(t) .
$$

The function $(t, x) \mapsto v^{-}(t)$ is clearly a subsolution of the equation (3.9) satisfied by $v$, with Neumann boundary conditions and with the initial condition $v^{-}(0) \leq v(0, x)$ on $\bar{\Omega}$. Thus, from the parabolic maximum principle, we have $v^{-}(t) \leq v(t, x)$ on $[0, T] \times \bar{\Omega}$. Similarly, since $v^{-}(t) \leq v(t, x)=\tilde{v}(t, x)$ on $[0, T] \times \partial \Omega$, the maximum principle implies that:

$$
0<r_{2} \leq v^{-}(t) \leq \tilde{v}(t, x) \text { on }[0, T] \times \bar{\Omega}
$$

The next lemma shows the boundedness of $\tilde{u}, \tilde{u}_{t}$ and $\tilde{u}_{t t}$ in well-suited spaces,

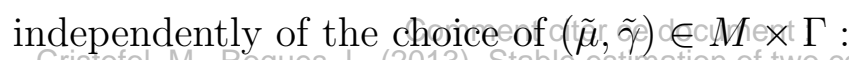


Lemma 3.3. For any $T>0$ it exists a constant $C>0$, independent of the choice of $(\tilde{\mu}, \tilde{\gamma}) \in M \times \Gamma$, such that:

$$
\|\tilde{u}\|_{C_{1}^{2}([0, T] \times \bar{\Omega})},\left\|\tilde{u}_{t}\right\|_{C_{1}^{2}([0, T] \times \bar{\Omega})},\left\|\tilde{u}_{t t}\right\|_{C_{1}^{2}([0, T] \times \bar{\Omega})} \leq C .
$$

Proof: First, from a classical comparison principle, we get

$$
0 \leq u, \tilde{u} \leq e^{T \max \mu^{+}} \max _{\bar{\Omega}} u_{i} \text {, on }[0, T] \times \bar{\Omega} .
$$

Next, we recall an a priori estimate for solutions of linear parabolic equations (see [44]):

Theorem 3.4. Assume that $f(t, x)$ is continuous on $[0, T] \times \bar{\Omega}$, with $f(0, \cdot)=0$ on $\partial \Omega$. Then, for any $\alpha \in(0,1)$, it exists a constant $C_{0}$, independent of $f$, such that any solution of

$$
\left\{\begin{array}{l}
\partial_{t} y-D \Delta y=f(t, x) \text { in } Q, \\
y=0 \text { in }(0, T) \times \partial \Omega \\
y(0, x)=0 \text { on } \bar{\Omega}
\end{array}\right.
$$

satisfies§

$$
\|y\|_{C_{0, \alpha / 2}^{1, \alpha}([0, T] \times \bar{\Omega})} \leq C_{0} \sup _{[0, T] \times \bar{\Omega}}|f| .
$$

We can apply Theorem 3.4 to $\tilde{y}=\tilde{u}-u$, in $(0, T] \times \Omega$, with $f(t, x)=\mu y-\gamma(u-$ $\tilde{u})(u+\tilde{u})+(\mu-\tilde{\mu}) \tilde{u}+(\gamma-\tilde{\gamma}) \tilde{u}^{2}$. Indeed, $f(0, x)=0$ on $\partial \Omega$ since $\mu=\tilde{\mu}$ and $\gamma=\tilde{\gamma}$ on $\partial \Omega$. Using (3.13) and the regularity of $u$ we thus obtain

$$
\|\tilde{u}\|_{C_{0, \alpha / 2}^{1, \alpha}([0, T] \times \bar{\Omega})} \leq C_{1}
$$

for some constant $C_{1}$ independent of the choice of $\tilde{\mu} \in M$ and of $\tilde{\gamma} \in \Gamma$.

Then, let us recall another result from parabolic regularity theory [44]:

Theorem 3.5. Assume that $g(t, x) \in C_{0, \alpha / 2}^{0, \alpha}([0, T] \times \bar{\Omega})$ and $h \in C_{1, \alpha / 2}^{2, \alpha}([0, T] \times \bar{\Omega})$ for some $\alpha \in(0,1)$, with the compatibility condition

$$
\partial_{t} h(0, x)-D \Delta h(0, x)=g(0, x) \text { for } x \in \partial \Omega .
$$

Then, it exists a constant $C_{0}^{\prime}$, independent of $g$ and $h$, such that the problem

$$
\left\{\begin{array}{l}
\partial_{t} z-D \Delta z=g(t, x) \text { in } Q \\
z(t, x)=h(t, x) \text { on }[0, T) \times \partial \Omega \cup(\{0\} \times \Omega),
\end{array}\right.
$$

has a unique solution $z \in C_{1, \alpha / 2}^{2, \alpha}([0, T] \times \bar{\Omega})$ which satisfies

$$
\|z\|_{C_{1, \alpha / 2}^{2, \alpha}([0, T] \times \bar{\Omega})} \leq C_{0}^{\prime}\left(\|g\|_{C_{0, \alpha / 2}^{0, \alpha}([0, T] \times \bar{\Omega})}+\|h\|_{C_{1, \alpha / 2}^{2, \alpha}([0, T] \times \bar{\Omega})}\right) .
$$

$\S$ The Hölder spaces $C_{j, \delta^{\prime}}^{i, \delta}([0, T] \times \bar{\Omega})$ are spaces of functions on $[0, T] \times \bar{\Omega}$ whose derivatives up to order $i$ in $x$ and order $j$ in $t$ are Hölder continuous with orders $\delta$ and $\delta^{\prime}$, respectively. 
Because of the regularity of $u$, we have $\partial_{t} u-D \Delta u=u(\mu-\gamma u)$ at $t=0$ on $\bar{\Omega}$. From the hypothesis on the coefficients and since $\tilde{u}=u=u_{i}$ at $t=0$, we get $\partial_{t} u-D \Delta u=\tilde{u}(\tilde{\mu}-\tilde{\gamma} \tilde{u})$ at $t=0$ on $\partial \Omega$. We can therefore apply Theorem 3.5, with $z=\tilde{u}, g=\tilde{u}(\tilde{\mu}-\tilde{\gamma} \tilde{u})$ and $h=u$, which gives:

$$
\|\tilde{u}\|_{C_{1, \alpha / 2}^{2, \alpha}([0, T] \times \bar{\Omega})} \leq C_{2}\left(\|\tilde{u}(\tilde{\mu}-\tilde{\gamma} \tilde{u})\|_{C_{0, \alpha / 2}^{0, \alpha}([0, T] \times \bar{\Omega})}+\|u\|_{C_{1, \alpha / 2}^{2, \alpha}([0, T] \times \bar{\Omega})}\right),
$$

and together with (3.16), this last inequality implies the existence of a constant $C_{3}$, independent of $\tilde{\mu}$ and $\tilde{\gamma}$, and such that:

$$
\|\tilde{u}\|_{C_{1, \alpha / 2}^{2, \alpha}([0, T] \times \bar{\Omega})} \leq C_{3} .
$$

Let us now set $v=\partial_{t} u$ and $\tilde{v}=\partial_{t} \tilde{u}$, as in the proof of Lemma 3.2. We have:

$$
\left\{\begin{array}{l}
\partial_{t} v=D \Delta v+v(\mu-2 \gamma u) \text { in }(0, T) \times \Omega, \\
\partial_{\nu} v=0 \text { on }[0, T) \times \partial \Omega \\
v(0, \cdot)=D \Delta u_{i}+u_{i}\left(\mu-\gamma u_{i}\right) \text { in } \Omega
\end{array}\right.
$$

and

$$
\left\{\begin{array}{l}
\partial_{t} \tilde{v}=D \Delta \tilde{v}+\tilde{v}(\tilde{\mu}-2 \tilde{\gamma} \tilde{u}) \text { in }(0, T) \times \Omega \\
\tilde{v}=v \text { on }[0, T) \times \partial \Omega \\
\tilde{v}(0, \cdot)=D \Delta u_{i}+u_{i}\left(\tilde{\mu}-\tilde{\gamma} u_{i}\right) \text { in } \Omega
\end{array}\right.
$$

Let us set

$$
g:=\tilde{v}(\tilde{\mu}-2 \tilde{\gamma} \tilde{u}) \text { and } h(t, x):=v(t, x)+\tilde{v}(0, x)-v(0, x) .
$$

Since $\mu=\tilde{\mu}$ and $\gamma=\tilde{\gamma}$ on $\partial \Omega$, we have

$$
\tilde{v}(0, x)-v(0, x)=0 \text { for } x \in \partial \Omega \text {. }
$$

Therefore, $h=v=\tilde{v}$ on $[0, T] \times \partial \Omega$, and since $h(0, x)=\tilde{v}(0, x)$ for $x \in \Omega$, we have $\tilde{v}=h$ for all $(t, x) \in[0, T] \times \partial \Omega \cup(\{0\} \times \Omega)$. Furthermore, at $t=0$,

$$
\partial_{t} h(0, x)-D \Delta h(0, x)=g(0, x) \text { on } \partial \Omega .
$$

Indeed, since $\mu(x)=\tilde{\mu}(x)$ and $\gamma(x)=\tilde{\gamma}(x)$ if $d(x, \partial \Omega)<\varepsilon$, we have, in addition to (3.24), $\Delta h(0, x)=\Delta \tilde{v}(0, x)=\Delta v(0, x)$ on $\partial \Omega$. As a consequence, (3.25) is equivalent to

$$
\partial_{t} v(0, x)-D \Delta v(0, x)=v(0, x)\left(\mu(x)-2 \gamma(x) u_{i}(x)\right) \text { on } \partial \Omega .
$$

This last equality is a consequence of (3.22) and of the regularity of $v: v \in C_{1}^{2}([0, T] \times \bar{\Omega})$.

We can therefore apply Theorem 3.5, to get:

$$
\|\tilde{v}\|_{C_{1, \alpha / 2}^{2, \alpha}([0, T] \times \bar{\Omega})} \leq C_{4}\left(\|g\|_{C_{0, \alpha / 2}^{0, \alpha}([0, T] \times \bar{\Omega})}+\|h\|_{C_{1, \alpha / 2}^{2, \alpha}([0, T] \times \bar{\Omega})}\right) .
$$

From the hypothesis on the coefficients and (3.21), we then obtain

$$
\|\tilde{v}\|_{C_{1, \alpha / 2}^{2, \alpha}([0, T] \times \bar{\Omega})} \leq C_{5}
$$

where $C_{5}$ is independent of $\tilde{\mu}$ and of $\tilde{\gamma}$. 
Setting $w=\partial_{t} v$ and $\tilde{w}=\partial_{t} \tilde{v}$, we can check that $w$ and $\tilde{w}$ are classical solutions of

$$
\left\{\begin{array}{l}
\partial_{t} w=D \Delta w+w(\mu-2 \gamma u)-2 \gamma v^{2} \text { in }(0, T) \times \Omega, \\
\partial_{\nu} w=0 \text { on }[0, T) \times \partial \Omega \\
w(0, \cdot)=D \Delta\left(\Delta u_{i}+u_{i}\left(\mu-\gamma u_{i}\right)\right)+\left(\Delta u_{i}+u_{i}\left(\mu-\gamma u_{i}\right)\right)\left(\mu-2 \gamma u_{i}\right) \text { in } \Omega .
\end{array}\right.
$$

and

$$
\left\{\begin{array}{l}
\partial_{t} \tilde{w}=D \Delta \tilde{w}+\tilde{w}(\tilde{\mu}-2 \tilde{\gamma} u)-2 \tilde{\gamma} \tilde{v}^{2} \text { in }(0, T) \times \Omega, \\
\tilde{w}=w \text { on }[0, T) \times \partial \Omega \\
\tilde{w}(0, \cdot)=D \Delta\left(\Delta u_{i}+u_{i}\left(\tilde{\mu}-\tilde{\gamma} u_{i}\right)\right)+\left(\Delta u_{i}+u_{i}\left(\tilde{\mu}-\tilde{\gamma} u_{i}\right)\right)\left(\tilde{\mu}-2 \tilde{\gamma} u_{i}\right) \text { in } \Omega .
\end{array}\right.
$$

The regularity of $w$ and $\tilde{w}$ - they belong to $C_{1}^{2}([0, T] \times \bar{\Omega})$ - follows from the assumption $u_{i} \in C^{7}(\bar{\Omega})$ and from the fact that $\mu(x)=\tilde{\mu}(x)$ and $\gamma(x)=\tilde{\gamma}(x)$ when $x$ is close to the boundary $\partial \Omega$.

Let us set

$$
g:=\tilde{w}(\tilde{\mu}-2 \tilde{\gamma} \tilde{u})-2 \tilde{\gamma} \tilde{v}^{2} \text { and } h(t, x):=w(t, x)+\tilde{w}(0, x)-w(0, x) .
$$

Using the same arguments as above with $w$ and $\tilde{w}$ instead of $v$ and $\tilde{v}$, we get

$$
\|\tilde{w}\|_{C_{1, \alpha / 2}^{2, \alpha}([0, T] \times \bar{\Omega})} \leq C_{6}\left(\|g\|_{C_{0, \alpha / 2}^{0, \alpha}([0, T] \times \bar{\Omega})}+\|h\|_{C_{1, \alpha / 2}^{2, \alpha}([0, T] \times \bar{\Omega})}\right) .
$$

From the hypothesis on the coefficients and (3.21) and (3.27), we get:

$$
\|\tilde{w}\|_{C_{1, \alpha / 2}^{2, \alpha}([0, T] \times \bar{\Omega})} \leq C_{7},
$$

where $C_{7}$ is independent of $\tilde{\mu}$ and of $\tilde{\gamma}$. Setting $C=\max \left\{C_{3}, C_{5}, C_{7}\right\}$, we obtain the result of Lemma 3.3 .

\section{Stability inequality}

\subsection{The inverse problem}

Let $u$ (resp. $\widetilde{u}$ ) be the solution of (2.3) (resp. of (2.4)) associated to $(\mu, \gamma)$ (resp. $(\tilde{\mu}, \tilde{\gamma}))$. We set $U=u-\widetilde{u}$. The function $U$ satisfies:

$$
\begin{cases}\partial_{t} U=D \Delta U+\mu U-\gamma U(\widetilde{u}+u)+\alpha \widetilde{u}-\beta \widetilde{u}^{2} & \text { in }(0, T) \times \Omega, \\ U(t, x)=0 & \text { on }[0, T) \times \partial \Omega, \\ U(0, x)=0 & \text { in } \Omega,\end{cases}
$$

where $\alpha=\mu-\tilde{\mu}$ and $\beta=\gamma-\tilde{\gamma}$. Using Lemma 3.2, part (i), we can set $y=U / \widetilde{u}$ and the previous system becomes:

$$
\left\{\begin{array}{rlrl}
\partial_{t} y= & D \Delta y+\frac{2 D}{\widetilde{u}} \nabla \widetilde{u} \cdot \nabla y & \\
& +y\left(\mu+D \frac{\Delta \widetilde{u}}{\widetilde{u}}-\frac{\partial_{t} \widetilde{u}}{\widetilde{u}}-\gamma(u+\tilde{u})\right)+\alpha-\beta \widetilde{u}, & & \text { in }(0, T] \times \Omega, \\
y(t, x)= & 0 & & \text { on }[0, T] \times \partial \Omega, \\
y(0, x)= & 0 & & \text { on } \Omega .
\end{array}\right.
$$


We set $z=\partial_{t} y$. Writing $A_{1}=\mu+D \Delta \widetilde{u} / \widetilde{u}-\partial_{t} \widetilde{u} / \widetilde{u}-\gamma(u+\tilde{u})$, we get:

$$
\left\{\begin{aligned}
\partial_{t} z= & D \Delta z+\frac{2 D}{\widetilde{u}} \nabla \widetilde{u} \cdot \nabla z+A_{1} z+\partial_{t}\left(\frac{2 D}{\widetilde{u}} \nabla \widetilde{u}\right) \cdot \nabla y & & \\
& +y \partial_{t} A_{1}-\beta \partial_{t} \widetilde{u}, & & \text { in }(0, T) \times \Omega, \\
z(t, x)= & 0 & & \text { on }[0, T) \times \partial \Omega, \\
z(0, x)= & \left(D \Delta y+\frac{2 D}{\widetilde{u}} \nabla \widetilde{u} \cdot \nabla y+A_{1} y(0, \cdot)+\alpha-\beta \widetilde{u}(0, \cdot)\right. & & \text { on } \Omega .
\end{aligned}\right.
$$

Then thanks to Lemma 3.2, part (ii), we set $\tilde{z}=z / \partial_{t} \tilde{u}, w=\partial_{t} \tilde{z}$ and for the sake of simplicity we denote:

$$
A_{2}=\left(-2 D \frac{\nabla \partial_{t} \tilde{u}}{\partial_{t} \tilde{u}}+2 D \frac{\nabla \tilde{u}}{\tilde{u}}\right)
$$

and

$$
A_{3}=\left(\frac{\partial_{t}^{2} \tilde{u}}{\partial_{t} \tilde{u}}-D \partial_{t} \tilde{u} \Delta\left(1 / \partial_{t} \tilde{u}\right)-2 D \frac{\nabla \partial_{t} \tilde{u} \cdot \nabla \tilde{u}}{\tilde{u} \partial_{t} \tilde{u}}+2 D\left|\nabla\left(1 / \partial_{t} \tilde{u}\right)\right|^{2}\left|\partial_{t} \tilde{u}\right|^{2}+A_{1}\right) .
$$

We obtain that $w$ satisfies the following system:

$$
\left\{\begin{array}{rlrl}
\partial_{t} w= & D \Delta w+A_{2} \cdot \nabla w+A_{3} w+2 D \partial_{t}\left(\frac{\nabla \tilde{u}}{\tilde{u}}\right) \cdot \frac{\nabla z}{\partial_{t} \tilde{u}}+\frac{\partial_{t} A_{1}}{\partial_{t} \tilde{u}} z & \\
& +\partial_{t}\left(A_{2}\right) \cdot \nabla \tilde{z}+\partial_{t}\left(A_{3}\right) \tilde{z}+\partial_{t}\left(2 D \partial_{t}\left(\frac{\nabla \tilde{u}}{\tilde{u}}\right) \frac{1}{\partial_{t} \tilde{u}}\right) \cdot \nabla y & \\
& +\partial_{t}\left(\frac{\partial_{t} A_{1}}{\partial_{t} \tilde{u}}\right) y & & \text { in }(0, T) \times \Omega, \\
w(t, x)= & 0 & & \text { on }[0, T) \times \partial \Omega .
\end{array}\right.
$$

Lemmas 3.2 and 3.3 show that all the quantities in the right-hand side of the equation satisfied by $w$ are well-defined.

\subsection{Carleman estimate}

In this section, we prove a Carleman inequality for the solution $w$ of the system (4.34).

Let $\omega$ be a nonempty subset of $\Omega$, included in the interior of $\Omega$. For any couple $0<\tau_{0}<\tau_{1}$ and for any $\delta \in\left(0, \tau_{0}\right)$, we set $Q_{\omega_{i}}=\left[\tau_{i}-\delta, \tau_{i}+\delta\right] \times \omega$ and $Q_{i}=\left[\tau_{i}-\delta, \tau_{i}+\delta\right] \times \Omega$, for $i=0,1$. Given any function $\zeta(x)$ satisfying:

$$
\zeta(x) \in C^{2}(\bar{\Omega}), \zeta(x)>0 \text { in } \Omega, \zeta(x)=0 \text { on } \partial \Omega, \text { and }|\nabla \zeta|>0 \text { on } \overline{\Omega \backslash \omega} \text {, }
$$

and any constant $K>0$, we may also define:

$$
\varphi_{i}(t, x)=\frac{e^{\lambda \zeta(x)}}{\left(t-\left(\tau_{i}-\delta\right)\right)\left(\tau_{i}+\delta-t\right)} \text { and } \eta_{i}(t, x)=\frac{e^{2 \lambda K}-e^{\lambda \zeta(x)}}{\left(t-\left(\tau_{i}-\delta\right)\right)\left(\tau_{i}+\delta-t\right)},
$$

where $\lambda>0$ and for $i=0,1$. Note that $\eta_{0}\left(\tau_{0}, \cdot\right)=\eta_{1}\left(\tau_{1}, \cdot\right)$ on $\bar{\Omega}$.

Let us recall a classical Carleman estimate (see [30]):

Theorem 4.1. Let $\tau_{0}, \tau_{1}, \delta$ such that $0<\tau_{0}<\tau_{1}$ and $0<\delta<\tau_{0}$. Then it exists a constant $K>0$, a function $\zeta(x)$ satisfying (4.35) and such that $K<\zeta(x)<2 K$, two constants $\lambda_{0} \geq 0, s_{0}>0$ and a positive constant $C_{0}$ such that, for any $\lambda \geq \lambda_{0}$, any 
$s \geq s_{0}$, and any function $q \in C^{2}\left(\overline{Q_{i}}\right)$ with $q \equiv 0$ on $\left[\tau_{i}-\delta, \tau_{i}+\delta\right] \times \partial \Omega$, the following estimate holds:

$$
\begin{aligned}
I_{i}(q) & \leq C_{0}\left[\int_{Q_{\omega_{i}}} e^{-2 s \eta_{i}} \lambda^{4}\left(s \varphi_{i}\right)^{3}|q|^{2} d t d x\right. \\
& \left.+\int_{Q_{i}} e^{-2 s \eta_{i}}\left|\partial_{t} q-D \Delta q\right|^{2} d t d x\right]
\end{aligned}
$$

where

$$
\begin{aligned}
I_{i}(q)= & \int_{Q_{i}} e^{-2 s \eta_{i}}\left(s \varphi_{i}\right)^{-1}\left(\left|\partial_{t} q\right|^{2}+|\Delta q|^{2}\right) d t d x \\
& +\lambda^{2} \int_{Q_{i}} e^{-2 s \eta_{i}} s \varphi_{i}|\nabla q|^{2} d t d x+\lambda^{4} \int_{Q_{i}} e^{-2 s \eta_{i}}\left(s \varphi_{i}\right)^{3}|q|^{2} d t d x .
\end{aligned}
$$

Remark 4.2. If we set

$$
M_{1}^{(i)} \psi=-D \Delta \psi-s^{2} \lambda^{2} D \varphi_{i}^{2}|\nabla \zeta|^{2} \psi+s \psi \partial_{t} \eta_{i}
$$

and

$$
M_{2}^{(i)} \psi=\partial_{t} \psi+2 s \lambda D \varphi_{i} \nabla \zeta \cdot \nabla \psi
$$

with $\psi=e^{-s \eta_{i}} q$, then the Carleman estimate (4.36) also gives an upper bound for $\left\|M_{1}^{(i)} \psi\right\|_{L^{2}\left(Q_{i}\right)}^{2}+\left\|M_{2}^{(i)} \psi\right\|_{L^{2}\left(Q_{i}\right)}^{2}($ see [46]).

Using Theorem 4.1 applied to the solution $y$ of (4.32), together with Lemma 3.2 and 3.3 we get that, for any $0<\tau_{0}<\tau_{1}, 0<\delta<\tau_{0}$ and for $s$ large enough, there exist $\vartheta(s)>0$ and $\kappa>0$ such that, independently of the choice of $(\tilde{\mu}, \tilde{\gamma}) \in M \times \Gamma$,

$$
I_{i}(y) \leq \vartheta(s) \int_{Q_{\omega_{i}}} \varphi_{i}^{3}|y|^{2} e^{-2 s \eta_{i}} d t d x+\kappa \int_{Q_{i}}|\alpha-\beta \tilde{u}|^{2} e^{-2 s \eta_{i}} d t d x .
$$

Note: in the sequel, for the sake of simplicity, we denote by $\kappa$ any constant independent of $s>0$ and of $(\tilde{\mu}, \tilde{\gamma}) \in M \times \Gamma$, and we denote by $\vartheta(s)$ any constant independent of $(\tilde{\mu}, \tilde{\gamma}) \in M \times \Gamma$.

We recall here the Lemma 2.1 of [47],

Lemma 4.3. Let $\tau_{i}, \delta, Q_{i}$ and $\eta_{i}$ be as previously. There exists a constant $\kappa=\kappa\left(\tau_{i}\right)>0$ depending only on $\tau_{i}$, and a constant $C(s)>0$, such that we have

$$
\int_{Q_{i}} \mathrm{e}^{-2 s \eta_{i}}\left|\int_{\tau_{i}}^{t} p(\xi, x) d \xi\right|^{2} d t d x \leq \frac{\kappa}{s}\left\|\mathrm{e}^{-s \eta_{i}} p\right\|_{\mathrm{L}^{2}\left(Q_{i}\right)}^{2}+C(s)\left\|\check{p}\left(\tau_{i}, .\right)\right\|_{L^{2}(\Omega)}^{2},
$$

for every $p \in \mathrm{L}^{2}\left(Q_{i}\right)$, all $s>0$ and with $\partial_{t} \check{p}=p$.

Now, using Theorem 4.1 applied to the solution $z$ of (4.33) and by Lemmas 3.3 and 4.3 , we get that there exist $\vartheta(s)>0$ and $\kappa>0$ such that :

$$
\begin{aligned}
I_{i}(z) & \leq \vartheta(s) \int_{Q_{\omega_{i}}} \varphi_{i}^{3}|z|^{2} e^{-2 s \eta_{i}} d t d x \\
& \quad \pm \kappa_{h} \int_{Q_{i}} \mid \beta d_{i t}^{2} e_{r}^{-2 s \eta_{i}} d t d t_{i} d x_{n}+\vartheta(s) \|\left(y\left(\tau_{i}, \cdot\right) \|_{H^{1}(\Omega)}^{2} .\right.
\end{aligned}
$$


Then, we are going to establish a similar inequality for $w$. We come back to the first equation of system (4.34) and we rewrite it in the following form:

$$
\partial_{t} w=D \Delta w+P_{1}(w)+P_{2}(z)+P_{3}(\tilde{z})+P_{4}(y)
$$

where $P_{i}(\cdot), i=1, \ldots, 4$ correspond to first order linear operators. First, by the classical Carleman estimate of Theorem 4.1 we have:

$$
\begin{aligned}
I_{i}(w) \leq & \kappa\left(\int_{Q_{i}} e^{-2 s \eta_{i}}\left|P_{1}(w)\right|^{2} d t d x+\int_{Q_{i}} e^{-2 s \eta_{i}}\left|P_{2}(z)\right|^{2} d t d x\right. \\
& \left.+\int_{Q_{i}} e^{-2 s \eta_{i}}\left|P_{3}(\tilde{z})\right|^{2} d t d x+\int_{Q_{i}} e^{-2 s \eta_{i}}\left|P_{4}(y)\right|^{2} d t d x\right) \\
& +\vartheta(s) \int_{Q_{\omega_{i}}} \varphi_{i}^{3}|w|^{2} e^{-2 s \eta_{i}} d t d x .
\end{aligned}
$$

From the definition of $I_{i}(w)$, see Eq. (4.37), the first integral is absorbed by the term $I_{i}(w)$ for $s$ large enough. Using Lemma 4.3, we observe that the second integral is bounded from above by $\frac{\kappa}{s} I_{i}(z)$ for $s$ large enough. Lemmas 3.2 and 3.3 imply that the third integral can be treated similarly. By Lemma 4.3, the fourth integral can be estimated as follows :

$$
\int_{Q_{i}}\left|P_{4}(y)\right|^{2} e^{-2 s \eta_{i}} d t d x \leq \frac{\kappa}{s} \int_{Q_{i}}\left(|z|^{2}+|\nabla z|^{2}\right) e^{-2 s \eta_{i}} d t d x+\vartheta(s)\left\|y\left(\tau_{i}, \cdot\right)\right\|_{H^{1}(\Omega)}^{2} .
$$

Finally, for $s$ large enough, we have:

$$
I_{i}(w) \leq \vartheta(s) \int_{Q_{\omega_{i}}} \varphi_{i}^{3}|w|^{2} e^{-2 s \eta_{i}} d t d x+\frac{\kappa}{s} I_{i}(z)+\vartheta(s) \|\left(y\left(\tau_{i}, \cdot\right) \|_{H^{1}(\Omega)}^{2},\right.
$$

and we get:

Theorem 4.4. Let $\tau_{0}, \tau_{1}, \delta$ be such that $0<\tau_{0}<\tau_{1}$ and $0<\delta<\tau_{0}$. Then there exist a constant $K>0$, a function $\zeta(x)$ in $C^{2}(\bar{\Omega})$ such that $K<\zeta(x)<2 K$, two constants $s_{1}>0$ and $\kappa>0$ such that, for any $s \geq s_{1}$, there exits $\vartheta(s)>0$ such that:

$$
\begin{aligned}
I_{i}(w) & \leq \vartheta(s) \int_{Q_{\omega_{i}}} e^{-2 s \eta_{i}} \varphi_{i}^{3}\left(|w|^{2}+|z|^{2}\right) d t d x+\frac{\kappa}{s} \int_{Q_{i}}|\gamma-\tilde{\gamma}|^{2} e^{-2 s \eta_{i}} d t d x \\
& +\vartheta(s) \|\left(y\left(\tau_{i}, \cdot\right) \|_{H^{1}(\Omega)}^{2} .\right.
\end{aligned}
$$

All the coefficients in this inequality are independent of $\tilde{\mu}$ and $\tilde{\gamma}$.

\subsection{Stability result}

Recall that our goal is to obtain a global stability estimate for both $\mu-\tilde{\mu}$ and $\gamma-\tilde{\gamma}$. A natural idea would be to obtain a first stability inequality for $\mu-\tilde{\mu}$, using the usual method in the case of a unique unknown coefficient, and then to obtain a second inequality for $\gamma-\tilde{\gamma}$. However, this approach faces two difficulties. First, the existing stability inequalities for one coefficient only work in the linear case. Second, even if these inequalities were available in the nonlinear context considered here, we would obtain two stability inequalities of athenform $\|\mu-\tilde{\mu}\| \leq C(\gamma) \| \operatorname{Observation}\left(\mathcal{S}_{\mu, \gamma}\right)-$ Cristofol, M., Roques, L. (2013). Stable estimation of two coefficie $\mu^{\prime}$
Fisher-KPP equation. Inverse Problems, 22 p. 
$\operatorname{Observation}\left(\mathcal{S}_{\tilde{\mu}, \gamma}\right) \|$, and $\|\gamma-\tilde{\gamma}\| \leq C(\mu)\left\|\operatorname{Observation}\left(\mathcal{S}_{\mu, \gamma}\right)-\operatorname{Observation}\left(\mathcal{S}_{\mu, \tilde{\gamma}}\right)\right\|$. These two inequalities are useless for the determination of $\mu$ and $\gamma$.

Here, we work at two different times $t_{0}$ and $t_{1}$ and, using Lemma 2.1, we adjust these values in order to get a single stability inequality for $\|\mu-\tilde{\mu}\|^{2}+\|\gamma-\tilde{\gamma}\|^{2}$.

In a first step, we get an upper bound for $\left\|\alpha e^{-s \eta_{0}\left(\tau_{0}, \cdot\right)}\right\|_{L^{2}(\Omega)}^{2}$, where $\alpha=\mu-\tilde{\mu}$ :

Lemma 4.5. Let $z$ and $w$ denote the solutions of (4.33) and (4.34), respectively. There exist three constants $s_{2}>0, \kappa>0$ and $\vartheta(s)>0$, such that:

$$
\begin{aligned}
\left\|\alpha e^{-s \eta_{0}\left(\tau_{0}, \cdot\right)}\right\|_{L^{2}(\Omega)}^{2} & \leq \vartheta(s) \int_{Q_{\omega_{0}}} \varphi_{0}^{3} e^{-2 s \eta_{0}}\left(|w|^{2}+|z|^{2}\right) d t d x+\vartheta(s)\left\|y\left(\tau_{0}, \cdot\right)\right\|_{H^{2}(\Omega)}^{2} \\
& +\left(2+\kappa / s^{2}\right) \max _{x \in \bar{\Omega}}\left|\tilde{u}\left(\tau_{0}, \cdot\right)\right|^{2}\left\|\beta e^{-s \eta_{0}\left(\tau_{0}, \cdot\right)}\right\|_{L^{2}(\Omega)}^{2}
\end{aligned}
$$

for all $s \geq s_{2}$.

Proof of Lemma 4.5. Let $\tau_{0}>0$ and $\delta \in\left(0, \tau_{0}\right)$. We begin with a technical lemma:

Lemma 4.6. There exists a constant $\tilde{s}>0$ depending only on $\tau_{0}$ such that, for all $q \in H^{1}\left(\left(\tau_{0}-\delta, \tau_{0}+\delta\right), L^{2}(\Omega)\right)$ and $s \geq \tilde{s}$ we have:

$$
\int_{\Omega}\left|q\left(\tau_{0}, x\right)\right|^{2} d x \leq 2\left(s \int_{Q_{0}}|q(t, x)|^{2} d t d x+s^{-1} \int_{Q_{0}}\left|\partial_{t} q(t, x)\right|^{2} d t d x t\right) .
$$

Proof of Lemma 4.6. Let $\chi \in \mathcal{C}^{\infty}(\mathbb{R} ;[0,1])$ be a cut-off function fulfilling

$$
\chi(t)= \begin{cases}1 & \text { if }\left|t-\tau_{0}\right|<\delta-2 \varepsilon, \\ 0 & \text { if }\left|t-\tau_{0}\right| \geq \delta-\varepsilon,\end{cases}
$$

for some fixed $\varepsilon \in(0, \delta / 2)$. Then, for every $q \in H^{1}\left(\left(\tau_{0}-\delta, \tau_{0}+\delta\right), L^{2}(\Omega)\right)$, the following identity holds:

$$
\begin{aligned}
& \int_{\Omega}\left|q\left(\tau_{0}, x\right)\right|^{2} d x=\int_{\tau_{0}-\delta}^{\tau_{0}} \frac{d}{d t}\left(\int_{\Omega} \chi^{2}(t)|q(t, x)|^{2} d x\right) d t \\
& =2\left(\int_{\tau_{0}-\delta}^{\tau_{0}} \int_{\Omega} \chi^{2}(t) q(t, x) \partial_{t} q(t, x) d t d x\right)+2 \int_{\tau_{0}-\delta}^{\tau_{0}} \int_{\Omega} \chi(t) \partial_{t} \chi(t)|q(t, x)|^{2} d t d x .
\end{aligned}
$$

Applying Young's inequality, this entails

$$
\int_{\Omega}\left|q\left(\tau_{0}, x\right)\right|^{2} d x \leq\left(s+2\left\|\partial_{t} \chi\right\|_{\infty}\right) \int_{Q_{0}}|q(t, x)|^{2} d t d x+s^{-1} \int_{Q_{0}}\left|\partial_{t} q(x, t)\right|^{2} d t d x,
$$

for each $s>0$, so the result follows by taking $\tilde{s}=2\left\|\partial_{t} \chi\right\|_{\infty}$.

Returning to the proof of Lemma 4.5, we consider the first equation of the system (4.32) evaluated at some time $t=\tau_{0}$ :

$z\left(\tau_{0}, \cdot\right)=\alpha-\beta \tilde{u}\left(\tau_{0}, \cdot\right)+\left(D \Delta y+\frac{2 D}{\widetilde{c}} \nabla \widetilde{u} \cdot \nabla y+y\left(\mu+D \frac{\Delta \widetilde{u}}{\widetilde{u}}-\frac{\partial_{t} \widetilde{u}}{\widetilde{u}}-\gamma(u+\tilde{u})\right)\right)\left(\tau_{0}, \cdot\right)$. 
Multiplying this equality by $e^{-s \eta_{0}\left(\tau_{0}, \cdot\right)}$ and applying Lemma 4.6 for $q=z e^{-s \eta_{0}}$, we get that

$$
\begin{aligned}
\int_{\Omega}\left|z\left(\tau_{0}, x\right)\right|^{2} e^{-2 s \eta_{0}\left(\tau_{0}, \cdot\right)} d x \leq & 3 s \int_{Q_{0}}|z(t, x)|^{2} e^{-2 s \eta_{0}(t, x)} d t d x \\
& +3 s^{-1} \int_{Q_{0}}\left|\partial_{t} z(t, x)\right|^{2} e^{-2 s \eta_{0}(t, x)} d t d x,
\end{aligned}
$$

for $s$ large enough. Using this last inequality and the equality satisfied by $z\left(\tau_{0}, \cdot\right)$, and using the results of Lemmas 3.2 and 3.3 we obtain:

$$
\begin{aligned}
\left\|\alpha e^{-s \eta_{0}\left(\tau_{0}, \cdot\right)}\right\|_{L^{2}(\Omega)}^{2} & \leq \kappa\left(s^{-1}\left\|w e^{-s \eta_{0}}\right\|_{L^{2}\left(\left(\tau_{0}-\delta, \tau_{0}+\delta\right), L^{2}(\Omega)\right)}^{2}\right. \\
& \left.\left.+s\left\|z e^{-s \eta_{0}}\right\|_{L^{2}\left(\left(\tau_{0}-\delta, \tau_{0}+\delta\right), L^{2}(\Omega)\right)}^{2}\right)+\left\|y\left(\tau_{0}, \cdot\right)\right\|_{H^{2}(\Omega)}^{2}\right) \\
& +2 \max _{x \in \bar{\Omega}}\left|\tilde{u}\left(\tau_{0}, \cdot\right)\right|^{2}\left\|\beta e^{-s \eta_{0}\left(\tau_{0}, \cdot\right)}\right\|_{L^{2}(\Omega)}^{2},
\end{aligned}
$$

for $s$ sufficiently large, and where $y$ is defined as in Section 4.1.

Using the inequality (4.39) and the result of Theorem 4.4 we can write:

$\left\|\alpha e^{-s \eta_{0}\left(\tau_{0}, \cdot\right)}\right\|_{L^{2}(\Omega)}^{2} \leq \kappa\left(\frac{I_{0}(w)}{s^{4}}+\frac{I_{0}(z)}{s^{2}}+\left\|y\left(\tau_{0}, \cdot\right)\right\|_{H^{2}(\Omega)}^{2}\right)+2 \max _{x \in \bar{\Omega}}\left|\tilde{u}\left(\tau_{0}, \cdot\right)\right|^{2}\left\|\beta e^{-s \eta_{0}\left(\tau_{0}, \cdot\right)}\right\|_{L^{2}(\Omega)}^{2}$,

and:

$$
\begin{aligned}
\left\|\alpha e^{-s \eta_{0}\left(\tau_{0}, \cdot\right)}\right\|_{L^{2}(\Omega)}^{2} & \leq \vartheta(s)\left(\int_{Q_{\omega_{0}}} \varphi_{0}^{3} e^{-2 s \eta_{0}}\left(|w|^{2}+|z|^{2}\right) d x d t+\left\|y\left(\tau_{0}, \cdot\right)\right\|_{H^{2}(\Omega)}^{2}\right) \\
& +\left(\kappa\left(\frac{1}{s^{5}}+\frac{1}{s^{2}}\right)+2 \max _{x \in \bar{\Omega}}\left|\tilde{u}\left(\tau_{0}, \cdot\right)\right|^{2}\right)\left\|\beta e^{-s \eta_{0}\left(\tau_{0}, \cdot\right)}\right\|_{L^{2}(\Omega)}^{2} .
\end{aligned}
$$

This concludes the proof of Lemma 4.5.

In a second step we give an upper bound for $\left\|\left(\alpha-\beta \tilde{u}\left(\tau_{1}, \cdot\right)\right) e^{-s \eta_{1}\left(\tau_{1}, \cdot\right)}\right\|_{L^{2}(\Omega)}^{2}$. The proof essentialy relies on the following Lemma

Lemma 4.7. Let $z$ denote the solution to (4.33) and put $\mathcal{I}:=\left\|\mathrm{e}^{-s \eta_{1}} z\left(\tau_{1}, \cdot\right)\right\|_{\mathrm{L}^{2}(\Omega)}^{2}$. Then there are three constants $s_{3}>0, \vartheta(s)>0$ and $\kappa>0$, such that we have

$$
\mathcal{I} \leq \vartheta(s) \int_{Q_{\omega_{1}}} e^{-2 s \eta_{1}}|z|^{2} d t d x+\kappa s^{-3 / 2} \int_{t_{1}-\delta}^{t_{1}} \int_{\Omega} e^{-2 s \eta_{1}}|\beta|^{2} d t d x
$$

for all $s \geq s_{3}$.

Proof of Lemma 4.7. Set $\psi=e^{-s \eta_{1}} z$. Bearing in mind that $\psi\left(\tau_{1}-\delta, \cdot\right)=0$ and since $\nabla \varphi_{1}=\lambda \varphi_{1} \nabla \zeta$, we find that

$$
\begin{aligned}
\mathcal{I} & =\int_{\tau_{1}-\delta}^{\tau_{1}} \int_{\Omega} \partial_{t}|\psi(t, x)|^{2} d t d x \\
& =2\left(\int_{\tau_{1}-\delta}^{\tau_{1}} \int_{\Omega} M_{2}^{(1)} \psi \psi d t d x-s D \int_{\tau_{1}-\delta}^{\tau_{1}} \int_{\Omega} \varphi_{1}|\nabla \zeta|^{2} \psi^{2} d x d t\right. \\
& \left.+s D \int_{\tau_{1}-\delta}^{\tau_{1}} \int_{\Omega} \varphi_{1} \Delta \zeta \psi^{2} d t d x\right) .
\end{aligned}
$$


Version définitive du manuscrit publiée dans / Final version of the manuscript published in :

Inverse problems (2013), 22 p., DOI: no data.

Stable estimation of two coefficients in a nonlinear Fisher-KPP equation

Then we get:

$$
\begin{aligned}
\int_{\Omega} e^{-2 s \eta_{1}\left(\tau_{1}, x\right)} z\left(\tau_{1}, x\right)^{2} d x & \leq 2\left|\int_{\tau_{1}-\delta}^{\tau_{1}} \int_{\Omega} M_{2}^{(1)} \psi \psi d t d x\right| \\
& +\kappa s \int_{\tau_{1}-\delta}^{\tau_{1}} \int_{\Omega} e^{-2 s \eta_{1}} \varphi_{1} z^{2} d t d x .
\end{aligned}
$$

Then we give an estimate of the first integral in the right hand side of the previous inequality. On the one hand from the Cauchy-Schwartz inequality:

$$
\left|\int_{\tau_{1}-\delta}^{\tau_{1}} \int_{\Omega} M_{2}^{(1)} \psi \psi d t d x\right| \leq s^{-3 / 2} \sqrt{\int_{\tau_{1}-\delta}^{\tau_{1}} \int_{\Omega}\left(M_{2}^{(1)} \psi\right)^{2} d t d x} \sqrt{s^{3} \int_{\tau_{1}-\delta}^{\tau_{1}} \int_{\Omega} e^{-2 s \eta_{1}} \varphi_{1}^{3} z^{2} d t d x} .
$$

Then using Young's inequality, we obtain

$$
\left|\int_{\tau_{1}-\delta}^{\tau_{1}} \int_{\Omega} M_{2}^{(1)} \psi \psi d t d x\right| \leq \frac{1}{2} s^{-3 / 2}\left(\left\|M_{2}^{(1)} \psi\right\|^{2}+s^{3} \int_{\tau_{1}-\delta}^{\tau_{1}} \int_{\Omega} e^{-2 s \eta_{1}} \varphi_{1}^{3} z^{2} d t d x\right)
$$

Finally by applying the Carleman inequality (4.39), we get:

$\left|\int_{\tau_{1}-\delta}^{\tau_{1}} \int_{\Omega} M_{2}^{(1)} \psi \psi d t d x\right| \leq \kappa\left[s^{3 / 2} \int_{\tau_{1}-\delta}^{\tau_{1}} \int_{\omega} e^{-2 s \eta_{1}} \varphi_{1}^{3} z^{2} d t d x+s^{-3 / 2} \int_{\tau_{1}-\delta}^{\tau_{1}} \int_{\Omega} e^{-2 s \eta_{1}}|\beta|^{2} d t d x\right]$.

Further by using inequality (4.45) and choosing $s$ sufficiently large, the following estimate yields:

$$
\mathcal{I} \leq \vartheta(s) \int_{Q_{\omega_{1}}} e^{-2 s \eta_{1}} \varphi_{1}^{3}|z|^{2} d t d x+\kappa s^{-3 / 2} \int_{\tau_{1}-\delta}^{\tau_{1}} \int_{\Omega} e^{-2 s \eta_{1}}|\beta|^{2} d x d t
$$

This concludes the proof of Lemma 4.7.

Let us come back to the first equation of the system (4.32) evaluated at some time $t=\tau_{1}$ :

$$
z\left(\tau_{1}, \cdot\right)=\left(D \Delta y+\frac{2}{\widetilde{u}} \nabla \widetilde{u} \cdot \nabla y+y\left(\mu+\frac{\Delta \widetilde{u}}{\widetilde{u}}-\frac{\partial_{t} \widetilde{u}}{\widetilde{u}}-\gamma(u+\tilde{u})\right)+\alpha-\beta \widetilde{u}\right)\left(\tau_{1}, \cdot\right) .
$$

Multiplying this equality by $e^{-s \eta_{1}\left(\tau_{1}, \cdot\right)}$ and combining with (4.46), we get:

Lemma 4.8. Let $z$ and $w$ denote the solutions of (4.33) and (4.34), respectively. Then there are three constants $s_{4}>0, \vartheta(s)>0$ and $\kappa>0$, such that we have

$$
\begin{aligned}
\left\|\left(\alpha-\beta \tilde{u}\left(\tau_{1}, \cdot\right)\right) e^{\left.-s \eta_{1}\left(\tau_{1}, \cdot\right)\right)}\right\|_{L^{2}(\Omega)}^{2} & \leq \vartheta(s) \int_{Q_{\omega_{1}}} e^{-2 s \eta_{1}}|z|^{2} d t d x \\
& +\vartheta(s)\left\|y\left(\tau_{1}, \cdot\right)\right\|_{H^{2}(\Omega)}^{2} \\
& +\kappa s^{-3 / 2} \int_{\tau_{1}-\delta}^{\tau_{1}} \int_{\Omega} e^{-2 s \eta_{1}}|\beta|^{2} d t d x,
\end{aligned}
$$

for all $s \geq s_{4}$. 
Version définitive du manuscrit publiée dans / Final version of the manuscript published in :

Inverse problems (2013), 22 p., DOI: no data.

Stable estimation of two coefficients in a nonlinear Fisher-KPP equation

It is straightforward that:

$$
\begin{aligned}
\left\|\alpha e^{\left.-s \eta_{1}\left(\tau_{1}, \cdot\right)\right)}\right\|_{L^{2}(\Omega)}^{2} & +\left\|\beta \tilde{u}\left(\tau_{1}, \cdot\right) e^{\left.-s \eta_{1}\left(\tau_{1}, \cdot\right)\right)}\right\|_{L^{2}(\Omega)}^{2} \\
& \leq 2\left\|\left(\alpha-\beta \tilde{u}\left(\tau_{1}, \cdot\right)\right) e^{\left.-s \eta_{1}\left(\tau_{1}, \cdot\right)\right)}\right\|_{L^{2}(\Omega)}^{2}+3\left\|\alpha e^{\left.-s \eta_{1}\left(\tau_{1}, \cdot\right)\right)}\right\|_{L^{2}(\Omega)}^{2} .
\end{aligned}
$$

Then since $\eta_{0}\left(\tau_{0}, \cdot\right)=\eta_{1}\left(\tau_{1}, \cdot\right)$ we get:

$$
\begin{aligned}
\left\|\alpha e^{\left.-s \eta_{0}\left(\tau_{0}, \cdot\right)\right)}\right\|_{L^{2}(\Omega)}^{2} & +\left\|\beta \tilde{u}\left(\tau_{1}, \cdot\right) e^{\left.-s \eta_{0}\left(\tau_{0}, \cdot\right)\right)}\right\|_{L^{2}(\Omega)}^{2} \\
& \leq 2\left\|\left(\alpha-\beta \tilde{u}\left(\tau_{1}, \cdot\right)\right) e^{\left.-s \eta_{1}\left(\tau_{1}, \cdot\right)\right)}\right\|_{L^{2}(\Omega)}^{2}+3\left\|\alpha e^{\left.-s \eta_{0}\left(\tau_{0}, \cdot\right)\right)}\right\|_{L^{2}(\Omega)}^{2} .
\end{aligned}
$$

and according to Lemmas 4.5 and 4.8 we obtain, for $s$ large enough:

$$
\begin{aligned}
\left\|\alpha e^{\left.-s \eta_{0}\left(\tau_{0}, \cdot\right)\right)}\right\|_{L^{2}(\Omega)}^{2} & +\left\|\beta \tilde{u}\left(\tau_{1}, \cdot\right) e^{\left.-s \eta_{0}\left(\tau_{0}, \cdot\right)\right)}\right\|_{L^{2}(\Omega)}^{2} \\
& \leq \vartheta(s) \int_{Q_{\omega_{1}}} \varphi_{1}^{3}|z|^{2} d t d x \\
& +\vartheta(s) \int_{Q_{\omega_{0}}} \varphi_{0}^{3}\left(|w|^{2}+|z|^{2}\right) d t d x \\
& +\vartheta(s)\left(\left\|y\left(\tau_{1}, \cdot\right)\right\|_{H^{2}(\Omega)}^{2}+\left\|y\left(\tau_{0}, \cdot\right)\right\|_{H^{2}(\Omega)}^{2}\right) \\
& +\left(\kappa / s^{2}+6 \max _{x \in \bar{\Omega}}\left|\tilde{u}\left(t_{0}, \cdot\right)\right|^{2}\right)\left\|\beta e^{-s \eta_{0}\left(t_{0}, \cdot\right)}\right\|_{L^{2}(\Omega)}^{2} .
\end{aligned}
$$

Thus, we get:

$$
\begin{aligned}
\left\|\alpha e^{\left.-s \eta_{0}\left(\tau_{0}, \cdot\right)\right)}\right\|_{L^{2}(\Omega)}^{2}+\left(\min _{x \in \bar{\Omega}}\left|\tilde{u}\left(t_{1}, x\right)\right|^{2}\right. & \left.-\frac{\kappa}{s^{2}}-6 \max _{x \in \bar{\Omega}}\left|\tilde{u}\left(t_{0}, \cdot\right)\right|^{2}\right)\left\|\beta \tilde{u}\left(\tau_{1}, \cdot\right) e^{\left.-s \eta_{0}\left(\tau_{0}, \cdot\right)\right)}\right\|_{L^{2}(\Omega)}^{2} \\
& \leq \vartheta(s) \int_{Q_{\omega_{1}}} \varphi_{1}^{3}|z|^{2} d t d x \\
& +\vartheta(s) \int_{Q_{\omega_{0}}} \varphi_{0}^{3}\left(|w|^{2}+|z|^{2}\right) d t d x \\
& +\vartheta(s)\left(\left\|y\left(\tau_{1}, \cdot\right)\right\|_{H^{2}(\Omega)}^{2}+\left\|y\left(\tau_{0}, \cdot\right)\right\|_{H^{2}(\Omega)}^{2}\right) .
\end{aligned}
$$

We then use Lemma 2.1 to find two times $t_{0}$ and $t_{1}$ such that

$$
6 \max _{x \in \bar{\Omega}} \tilde{u}\left(t_{0}, x\right)<\min _{x \in \bar{\Omega}} \tilde{u}\left(t_{1}, x\right)
$$

and we fix $\delta \in\left(0, t_{0}\right)$. Applying the inequality (4.48) with $\tau_{0}=t_{0}$ and $\tau_{1}=t_{1}$ and $s$ large enough, we obtain the existence of a constant $C>0$ such that, for any couple $(\tilde{\mu}, \tilde{\gamma}) \in M \times \Gamma$

$$
\begin{aligned}
\|\alpha\|_{L^{2}(\Omega)}^{2}+\|\beta\|_{L^{2}(\Omega)}^{2} & \leq C \int_{t_{0}-\delta}^{t_{1}+\delta} \int_{\omega}\left(|z|^{2}+|w|^{2}\right) d t d x+C\left\|y\left(t_{0}, \cdot\right)\right\|_{H^{2}(\Omega)}^{2} \\
& +C\left\|y\left(t_{1}, \cdot\right)\right\|_{H^{2}(\Omega)}^{2} \\
& \leq C\left(\|u-\tilde{u}\|_{\left.H^{2}\left(t_{0}-\delta, t_{1}+\delta\right), L^{2}(\omega)\right)}^{2}+\left\|(u-\tilde{u})\left(t_{0}, \cdot\right)\right\|_{H^{2}(\Omega)}^{2}\right. \\
& \left.+\left\|(u-\tilde{u})\left(t_{1}, \cdot\right)\right\|_{H^{2}(\Omega)}^{2}\right)
\end{aligned}
$$

and Theorem 2.2 follows directly, ce document 


\section{Appendix A: existence of non-constant initial conditions satisfying (2.5) and (2.6)}

Under our assumptions, we can prove the existence of a non-constant function $u_{i}$ which satisfies (2.5) and (2.6). Let us recall that, from Krein-Rutman theory (see [48] for further details), for any $\rho \in C^{5}(\bar{\Omega})$, it exists a unique real number $\lambda_{1}[\rho]$ and a unique function $\varphi \in C^{7}(\bar{\Omega})$ which satisfy

$$
\left\{\begin{array}{l}
-D \Delta \varphi-\rho \varphi=\lambda_{1}[\rho] \varphi \text { in } \Omega \\
\partial_{\nu} \varphi=0 \text { on } \partial \Omega, \varphi>0 \text { in } \Omega,\|\varphi\|_{\infty}=1 .
\end{array}\right.
$$

Definition 4.9. $\lambda_{1}[\rho]$ and $\varphi$ are respectively the principal eigenvalue and principal eigenfunction of the operator $-D \Delta-\rho$, with Neumann boundary conditions.

Let us set $\lambda^{-}=\lambda_{1}\left[\mu^{-}\right]$, and let $\varphi^{-}$be the associated principal eigenfunction.

Lemma 4.10. For any $\kappa \in\left(0, \frac{\min _{\bar{\Omega}} \mu^{-}}{6 \max _{\bar{\Omega}} \gamma^{+}}\right)$, the function $u_{i}=\kappa \varphi^{-}$satisfies (2.5) and (2.6).

Proof. Let us first show that $\lambda^{-}<0$. We have

$$
-D \Delta \varphi^{-}-\mu^{-} \varphi^{-}=\lambda^{-} \varphi^{-} \text {in } \Omega \text {. }
$$

Dividing this equation by $\varphi$ and integrating by parts over $\Omega$, we get:

$$
-D \int_{\Omega} \frac{\left|\nabla \varphi^{-}\right|^{2}}{\left(\varphi^{-}\right)^{2}}-\int_{\Omega} \mu^{-}=\lambda^{-}|\Omega|,
$$

where $|\Omega|$ stands for the Lebesgue measure of the set $\Omega$. Using our assumption $\mu^{-}>0$ in $\Omega$, we get

$$
\lambda^{-}<0 .
$$

Now, let us set $u_{i}=\kappa \varphi^{-}$, for some $\kappa>0$. The function $u_{i}$ satisfies

$$
-D \Delta u_{i}-u_{i}\left(\mu^{-}-\gamma^{+} u_{i}\right)=u_{i}\left(\lambda^{-}+\gamma^{+} \kappa \varphi^{-}\right) \text {in } \Omega .
$$

In particular, if $\kappa \in\left(0, \frac{-\lambda^{-}}{\max _{\bar{\Omega}} \gamma^{+}}\right)$, we obtain

$$
-D \Delta u_{i}-u_{i}\left(\mu^{-}-\gamma^{+} u_{i}\right)<0 \text { in } \Omega,
$$

and the condition $(2.6)$ is fulfilled. Since $\max _{\bar{\Omega}} u_{i}=\kappa$, the assumption (2.5) is also satisfied if $\kappa<\min _{\bar{\Omega}} \mu^{-} /\left(6 \max _{\bar{\Omega}} \gamma^{+}\right)$. Since $-\lambda^{-}>\min _{\bar{\Omega}} \mu^{-}$(this can easily be checked by using Rayleigh formula), $u_{i}=\kappa \varphi^{-}$satisfies the assumptions (2.5) and (2.6) for any $\kappa \in\left(0, \frac{\min _{\bar{\Omega}} \mu^{-}}{6 \max _{\bar{\Omega}} \gamma^{+}}\right)$. 


\section{Appendix B: Proof of Lemma 2.1}

We recall that we want to prove that it exists a bounded interval $\mathcal{T} \subset(0, \infty)$ such that, for any couple $\left(t_{0}, t_{1}\right)$ with $0<t_{0} \leq \inf \mathcal{T}<\sup \mathcal{T} \leq t_{1}$, and for all $(\mu, \gamma)$ and $(\tilde{\mu}, \tilde{\gamma}) \in M \times \Gamma$

$$
6 \max _{x \in \bar{\Omega}} \tilde{u}\left(t_{0}, x\right)<\min _{x \in \bar{\Omega}} \tilde{u}\left(t_{1}, x\right) .
$$

The proof of this lemma uses the hypothesis (2.5) on $u_{i}$.

Recall the definition of the sets $M$ and $\Gamma$ :

$$
M:=\left\{\tilde{\mu} \in C^{5}(\bar{\Omega}) \text { s.t. } \mu^{-} \leq \tilde{\mu} \leq \mu^{+} \text {on } \bar{\Omega} \text { and }\|\tilde{\mu}\|_{C^{5}(\bar{\Omega})} \leq m\right\}
$$

and

$$
\Gamma:=\left\{\tilde{\gamma} \in C^{5}(\bar{\Omega}) \text { s.t. } \gamma^{-} \leq \tilde{\gamma} \leq \gamma^{+} \text {on } \bar{\Omega} \text { and }\|\tilde{\gamma}\|_{C^{5}(\bar{\Omega})} \leq m\right\} .
$$

Let us fix two couples $(\mu, \gamma)$ and $(\tilde{\mu}, \tilde{\gamma})$ in $M \times \Gamma$, and a function $u_{i}$ satisfying the hypothesis (2.5). Let $u$ and $\tilde{u}$ be respectively the solutions of (2.3) and (2.4).

Let us set

$$
\underline{\mu}^{-}=\min _{\bar{\Omega}}\left\{\mu^{-}\right\}, \bar{\mu}^{+}=\max _{\bar{\Omega}}\left\{\mu^{+}\right\} \text {and } \bar{\gamma}^{+}=\max _{\bar{\Omega}}\left\{\gamma^{+}\right\}
$$

and similarly,

$$
\underline{u_{i}}=\min _{\bar{\Omega}}\left\{u_{i}\right\} \text { and } \overline{u_{i}}=\max _{\bar{\Omega}}\left\{u_{i}\right\}
$$

Let $u^{-}$and $u^{+}$be defined as in the proof of Lemma 3.2:

$$
\left\{\begin{array}{llrl}
\frac{d u^{-}}{d t} & =u^{-}\left(\underline{\mu}^{-}-\bar{\gamma}^{+} u^{-}\right), & & u^{-}(0)=\underline{u_{i}} \\
\frac{d u^{+}}{d t} & =u^{+} \bar{\mu}^{+}, & & u^{+}(0)=\overline{u_{i}}
\end{array}\right.
$$

The functions $(t, x) \mapsto u^{-}(t)$ and $(t, x) \mapsto u^{+}(t)$ are respectively sub- and supersolutions of the equation satisfied by $u$, with Neumann boundary conditions and with $u^{-}(t) \leq$ $u(t, x) \leq u^{+}(t)$ on $\bar{\Omega}$ at $t=0$. We therefore have

$$
u^{-}(t) \leq u(t, x) \leq u^{+}(t) \text { for all } t \geq 0, x \in \bar{\Omega}
$$

In particular we have $u^{-}(t) \leq \tilde{u}(t, x) \leq u^{+}(t)$ in $\{0\} \times \bar{\Omega} \cup(0,+\infty) \times \partial \Omega$, and since $(t, x) \mapsto u^{-}(t)$ and $(t, x) \mapsto u^{+}(t)$ are also sub- and supersolutions of the equation satisfied by $\tilde{u}$, we get

$$
u^{-}(t) \leq \tilde{u}(t, x) \leq u^{+}(t) \text { for all } t \geq 0, x \in \bar{\Omega}
$$

Fix some times $t_{0}, t_{1}$, with $t_{0}<t_{1}$. We have

$$
u^{+}\left(t_{0}\right)=\overline{u_{i}} e^{\bar{\mu}^{+} t_{0}} \text { and } u^{-}\left(t_{1}\right)=\frac{\underline{\mu}^{-} \underline{u}_{i} e^{\underline{\mu}^{-} t_{1}}}{\underline{\mu}^{-}+\bar{\gamma}^{+} \underline{u_{i}}\left(e^{\underline{\mu}^{-} t_{1}}-1\right)} .
$$


Version définitive du manuscrit publiée dans / Final version of the manuscript published in :

Inverse problems (2013), 22 p., DOI: no data.

Stable estimation of two coefficients in a nonlinear Fisher-KPP equation

Thus, it exists a couple $\left(\tau_{0}, \tau_{1}\right)$ such that $0<\tau_{0}<\tau_{1}$ and $u^{-}\left(\tau_{1}\right)>6 u^{+}\left(\tau_{0}\right)$ if and only if $\frac{\mu^{-}}{\bar{\gamma}^{+}}>6 \overline{u_{i}}$, which corresponds to the assumption $(2.5)$ on $u_{i}$. For such a couple $\left(\tau_{0}, \tau_{1}\right)$ we then get, for all $t_{0} \leq \tau_{0}$, and $t_{1} \geq \tau_{1}$,

$$
6 \max _{x \in \bar{\Omega}} \tilde{u}\left(t_{0}, x\right) \leq 6 u^{+}\left(t_{0}\right) \leq 6 u^{+}\left(\tau_{0}\right)<u^{-}\left(\tau_{1}\right) \leq u^{-}\left(t_{1}\right) \leq \min _{x \in \bar{\Omega}} \tilde{u}\left(t_{1}, x\right) .
$$

Note that the formula (4.55) allows an explicit computation of the the interval $\mathcal{T}=\left[\tau_{0}, \tau_{1}\right]$. 
Version définitive du manuscrit publiée dans / Final version of the manuscript published in :

Inverse problems (2013), 22 p., DOI: no data.

Stable estimation of two coefficients in a nonlinear Fisher-KPP equation

\section{References}

[1] P Grindrod. Theory and Applications of Reaction-Diffusion Equations. Clarendon Press, 1996.

[2] A Okubo and S A Levin. Diffusion and ecological problems - modern perspectives. Second edition, Springer-Verlag, New York, 2002.

[3] N Shigesada and K Kawasaki. Biological Invasions: Theory and Practice. Oxford Series in Ecology and Evolution, Oxford: Oxford University Press, 1997.

[4] $\mathrm{P}$ Turchin. Quantitative analysis of movement: measuring and modeling population redistribution in animals and plants. Sinauer, Sunderland, MA, 1998.

[5] D G Aronson and H G Weinberger. Nonlinear diffusion in population genetics, combustion and nerve propagation. In Partial Differential Equations and Related Topics, volume 446 of Lectures Notes Math, pages 5-49. Springer, New York, 1975.

[6] D G Aronson and H G Weinberger. Multidimensional nonlinear diffusion arising in population genetics. Adv. Math., 30(1):33-76, 1978.

[7] R A Fisher. The wave of advance of advantageous genes. Ann Eugen, 7:335-369, 1937.

[8] A N Kolmogorov, I G Petrovsky, and N S Piskunov. Étude de l'équation de la diffusion avec croissance de la quantité de matière et son application à un problème biologique. Bull. Univ. État Moscou, Sér. Int. A, 1:1-26, 1937.

[9] L Roques, J Garnier, F Hamel, and E K Klein. Allee effect promotes diversity in traveling waves of colonization. Proc. Natl. Acad. Sci. USA, 109(23):8828-8833, 2012.

[10] J Billingham and D J Needham. The development of traveling waves in quadratic and cubic autocatalysis with unequal diffusion rates. I. Permanent form of traveling waves. Phil. Trans. Royal Soc. A, 334:1-24, 1991.

[11] P C Fife. Mathematical Aspects of Reacting and Diffusing Systems, volume 28 of Lecture Notes in Biomathematics. Springer-Verlag, 1979.

[12] H Berestycki and B Larrouturou. Quelques aspects mathématiques de la propagation des flammes prémélangées, volume 10 of Collège de France Seminar, pages 65-129. Pitman Longman, Harbow, UK, 1990.

[13] J G Skellam. Random dispersal in theoretical populations. Biometrika, 38:196-218, 1951.

[14] S Cantrell, R and C Cosner. Spatial ecology via reaction-diffusion equations. John Wiley \& Sons Ltd, Chichester, UK, 2003.

[15] L Roques and R S Stoica. Species persistence decreases with habitat fragmentation: an analysis in periodic stochastic environments. Journal of Mathematical Biology, 55(2):189-205, 2007.

[16] H Berestycki, F Hamel, and L Roques. Analysis of the periodically fragmented environment model: I - Species persistence. Journal of Mathematical Biology, 51(1):75-113, 2005.

[17] H Berestycki, F Hamel, and L Roques. Analysis of the periodically fragmented environment model: II - Biological invasions and pulsating travelling fronts. Journal de Mathematiques Pures et Appliquées, 84(8):1101-1146, 2005.

[18] M El Smaily, F Hamel, and L Roques. Homogenization and influence of fragmentation in a biological invasion model. Discrete and Continuous Dynamical Systems, Series A, 2009.

[19] F Hamel, J Fayard, and L Roques. Spreading speeds in slowly oscillating environments. Bull Math Biol, 72(5):1166-1191, 2010.

[20] N Shigesada, K Kawasaki, and E Teramoto. Traveling periodic-waves in heterogeneous environments. Theor Popul Biol, 30(1):143-160, 1986.

[21] J Xin. Front propagation in heterogeneous media. SIAM Review, 42:161-230, 2000.

[22] DAISIE. Handbook of alien species in Europe. Springer, Dordrecht, 2009.

[23] L Roques, S Soubeyrand, and J Rousselet. A statistical-reaction-diffusion approach for analyzing expansion processes. Journal of Theoretical Biology, 274:43-51, 2011.

[24] F Hamel, G Nadin, and L Roques. A viscosity solution method for the spreading speed formula in slowly varying media. Indiana Univ. Math. J., In press, 60:1229-1247, 2011.

[25] H Tong. Non-linear Time Series: A Dynamical System Approach. Oxford University Press, 1990. 
Version définitive du manuscrit publiée dans / Final version of the manuscript published in :

Inverse problems (2013), 22 p., DOI: no data.

Stable estimation of two coefficients in a nonlinear Fisher-KPP equation

[26] C K Wikle. Hierarchical models in environmental science. International Statistical Review, 71:181199, 2003.

[27] M V de Hoop, L Qiu, and O Scherzer. Local analysis of inverse problems: Höder stability and iterative reconstruction. Inverse Problems, 28:045001, 2012.

[28] M Belassoued and M Yamamoto. Inverse source problem for a transmission problem for a parabolic equation. Journal of Inverse and Ill-Posed Problems, 14(1):47-56, 2006.

[29] M Cristofol and L Roques. Biological invasions: Deriving the regions at risk from partial measurements. Math Biosci, 215(2):158-166, 2008.

[30] O Y Immanuvilov and M Yamamoto. Lipschitz stability in inverse parabolic problems by the Carleman estimate. Inverse Problems, 14:1229-1245, 1998.

[31] M Yamamoto and J Zou. Simultaneous reconstruction of the initial temperature and heat radiative coefficient. Inverse Problems, 17:1181-1202, 2001.

[32] A L Bukhgeim and M V Klibanov. Uniqueness in the large of a class of multidimensional inverse problems. Soviet Mathematics - Doklady, 24:244-247, 1981.

[33] M Yamamoto. Carleman estimates for parabolic equations and applications. Inverse Problems, 25(12):123013, 2009.

[34] M Cristofol, J Garnier, F Hamel, and L Roques. Uniqueness from pointwise observations in a multi-parameter inverse problem. Communications on Pure and Applied Analysis, 11:1-15, 2011.

[35] L Roques and M Cristofol. On the determination of the nonlinearity from localized measurements in a reaction-diffusion equation. Nonlinearity, 23:675-686, 2010.

[36] L Roques and M Cristofol. The inverse problem of determining several coefficients in a nonlinear lotka-volterra system. Inverse Problems, 28:075007, 2012.

[37] M Choulli, E M Ouhabaz, and M Yamamoto. Stable determination of a semilinear term in a parabolic equation. Communications on Pure and Applied Analysis, 5:447-462, 2006.

[38] P DuChateau and W Rundell. Unicity in an inverse problem for an unknown reaction term in a reaction-diffusion equation. Journal of Differential Equations, 59:155-164, 1985.

[39] H Egger, H W Engl, and M Klibanov. Global uniqueness and Hölder stability for recovering a nonlinear source term in a parabolic equation. Inverse Problems, 21:271-290, 2005.

[40] A Lorenzi. An inverse problem for a semilinear parabolic equation. Annali di Matematica Pura ed Applicata, 131:145-166, 1982.

[41] S Nakamura. A note on uniqueness in an inverse problem for a semilinear parabolic equation. Nihonkai Mathematical Journal, 12:71-73, 2001.

[42] M S Pilant and W Rundell. An inverse problem for a nonlinear parabolic equation. Communications in Partial Differential Equations, 11:445-457, 1986.

[43] C V Pao. Nonlinear Parabolic and Elliptic Equations. Plenum Press, New York, 1992.

[44] A Friedman. Partial differential equations of parabolic type. Prentice-Hall, Englewood Cliffs, NJ, 1964.

[45] L C Evans. Partial differential equations. University of California, Berkeley - AMS, 1998.

[46] A Fursikov. Optimal control of distribued systems, volume 187 of Translations of Mathematical Monographs. American Mathematical Society, Providence, RI, 2000.

[47] M V Klibanov. Global uniqueness of a multidimensional inverse problem for a nonlinear parabolic equation by a Carleman estimate. Inverse Problems, 20:1003-1032, 2004.

[48] D Gilbarg and N S Trudinger. Elliptic Partial Differential Equations of Second Order. SpringerVerlag, Berlin, 1983. 